\title{
Shortened antibody screening interval has decreased the incidence of delayed hemolytic transfusion reactions
}

\author{
Nozomi Takano, Hiroyasu Yasuda, Masami Kikuchi, Kinuyo Kawabata, \\ Chikako Takeuchi-Baba, Satoshi Ono, Takako Ono, Keiji Minakawa, \\ Akiko Sugawara, Saori Miura, Kazuya Watanabe, Maiko Abe, \\ Hiroe Suzuki, Mao Watanabe, Mutsumi Sasaki, Kazuhiko Ikeda, \\ Kenneth E. Nollet, Hitoshi Ohto
}

\begin{abstract}
Aims: To ascertain the effects of allowable sampling time prior to red cell transfusion when screening for alloantibodies, we compared antibody detection rates and frequencies of delayed hemolytic transfusion reactions (DHTRs) among patients drawn within two weeks versus patients drawn within one week of transfusion. Methods: Alloantibody screening for 32,601 patients from January 1997 through August 2006 was done within two weeks of transfusion, and for 44,896 patients from September 2006 through March 2017 was done within one week. Among transfusion recipients, 6,234 screened within two weeks and 8,066 screened within one week were evaluated for DHTR. Results: Alloantibodies were detected in $1.2 \%$ of cases screened within two weeks and in $1.3 \%$ of cases
\end{abstract}

Nozomi Takano ${ }^{1}$, Hiroyasu Yasuda ${ }^{1}$, Masami Kikuchi ${ }^{1}$ Kinuyo Kawabata ${ }^{1}$, Chikako Takeuchi-Baba' ${ }^{1}$ Satoshi Ono ${ }^{1}$ Takako Ono', Keiji Minakawa', Akiko Sugawara', Saori Miura $^{1}$, Kazuya Watanabe ${ }^{1}$, Maiko Abe ${ }^{1}$, Hiroe Suzuki ${ }^{1}$, Mao Watanabe $^{1}$, Mutsumi Sasaki ${ }^{1}$, Kazuhiko Ikeda ${ }^{1}$, Kenneth E. Nollet $^{1}$, Hitoshi Ohto ${ }^{1,2}$

Affiliations: ${ }^{1}$ Department of Blood Transfusion and Transplantation Immunology, Fukushima Medical University Hospital, Fukushima, Japan; ${ }^{2}$ Department of Advanced Cancer Immunotherapy, Fukushima Medical University, Fukushima, Japan.

Corresponding Author: Hitoshi Ohto, MD, PhD, Department of Blood Transfusion and Transplantation Immunology, Fukushima Medical University School of Medicine, Fukushima City, Fukushima 960-1295, Japan; Email: hit-ohto@ fmu.ac.jp

Received: 03 May 2018

Accepted: 12 June 2018

Published: 23 June 2018 screened within one week, with no statistical difference. Anti-Fy ${ }^{\mathrm{b}}$ was more frequently detected (115.8 versus 39.9 per 100,000 patients) among those screened within one week $(p<0.001)$; anti$\mathrm{Di}^{\mathrm{a}}$ showed a similar trend, but without statistical significance $(p=0.065)$, whereas anti-c was less frequently detected $(p<0.05)$. The number of DHTRs observed was significantly lower among those screened within one week, with two cases (1 in 4,031) versus eight ( 1 in 779 ) among those screened within 2 weeks $(p<0.05)$. Conclusion: By shortening allowable sampling period for red cell alloantibody screening from within two weeks to within one week of transfusion, the detection rate of clinically significant antibodies, except for anti-c, increased, and the frequency of DHTR recipients decreased.

Keywords: Alloantibody, Clinically significant antibody, Delayed hemolytic transfusion reaction, Irregular antibody screening

\section{How to cite this article}

Takano N, Yasuda H, Kikuchi M, Kawabata K, TakeuchiBaba C, Ono S, Ono T, Minakawa K, Sugawara A, Miura S, Watanabe K, Abe M, Suzuki H, Watanabe M, Sasaki M, Ikeda K, Nollet KE, Ohto H. Shortened antibody screening interval has decreased the incidence of delayed hemolytic transfusion reactions. Int J Blood Transfus Immunohematol 2018;8:100042Z02NT2018.

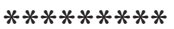

Article ID: 100042Z02NT2018

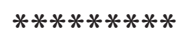

doi: 10.5348/100042Zo2NT2018OA 


\section{EDORIUM Journals}

Int J Blood Transfus Immunohematol 2018;8:100042Z02NT2018. www.ijbti.com

Takano et al. 2

\section{INTRODUCTION}

Red blood cell (RBC) alloantibody screening is essential to prevent acute and delayed hemolytic transfusion reactions (DHTRs); any failure to detect alloantibodies places a recipient at risk. Early screening might precede alloantibody induction and late screening might miss alloantibody levels that have fallen below detection sensitivity [1]. Guidelines are intended to balance high detection sensitivity against the practical matter of being able to find units that are serologically and physiologically compatible [2].

In the UK, the British Committee for Standards in Haematology (BCSH) recommends that serological studies use patient samples collected no more than 3 days prior to the actual transfusion if a patient has been transfused or pregnant within the preceding 3 months, with allowance for samples up to 7 days prior for patients with no alloantibody history, no recent transfusion, and no recent pregnancy [3]. AABB and Dutch Blood Transfusion Guidelines also recommend antibody screening no more than 3 days before red cell transfusion for a patient who has been pregnant or transfused within the previous three months $[4,5]$, whereas the Canadian Society for Transfusion Medicine advises that specimens be collected within 96 hours of transfusion [3].

BCSH acknowledges, however, that scientific evidence is limited, and that their policy should be considered as a baseline only [3]. Thus, in this study, we aspired to analyze a large body of historical data in which differences might emerge between patients screened within 2 weeks versus 1 week of red cell transfusion. Any such differences might inform future pre-transfusion guidelines.

\section{MATERIALS AND METHODS}

\section{Patients}

Patients were screened for alloantibodies using samples obtained within 2 weeks $(2 \mathrm{~W})$ or 1 week $(1 \mathrm{~W})$, according to policies in place from January 1997 through August 2006 (9.6 years) and September 2006 through March 2017 (10.5years), respectively. For cross-matching, samples collected 72 hours prior to RBC transfusion were used. Groups 2W and 1W included 32,601 and 44,896 patients, respectively, of whom 6,234 and 8,066 actually received $\mathrm{RBC}$ transfusion (Figure 1).

\section{Pre-transfusion serological tests}

Alloantibody screening and identification was performed by a standard tube technique $[6,7]$. Four-cell panels comprised of Surgiscreen (three cells) and $\mathrm{Di}^{\mathrm{a}}$ (bothOrtho Clinical Diagnostics, NJ, USA), were used for antibody screening. In saline method, 2 drops of patient's serum or plasma and 1 drop of each of screen cells were mixed, incubated at room temperature for 10 minutes (Sal-RT10') during the $2 \mathrm{~W}$ period or without incubation

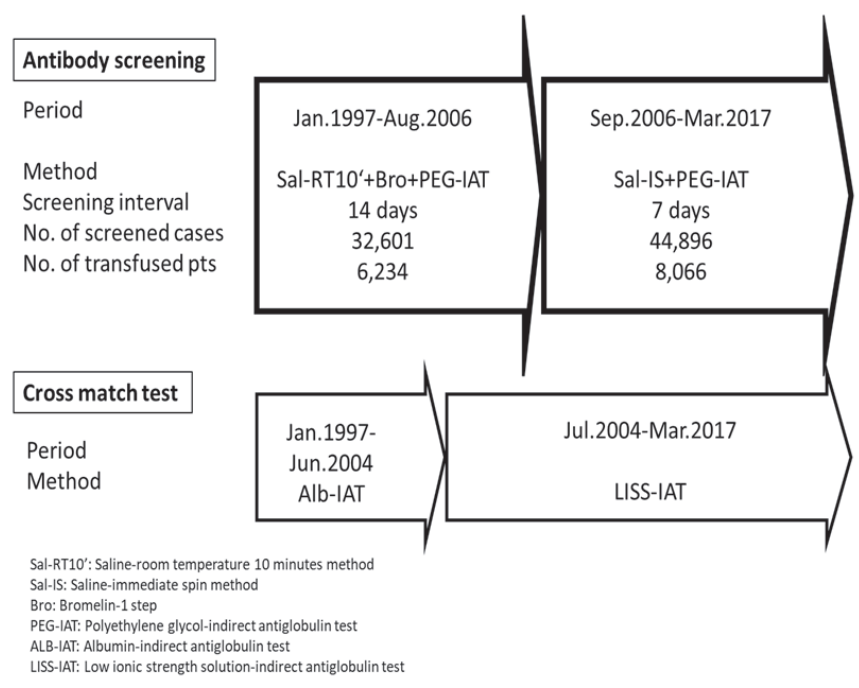

Figure 1: Time course of the study periods.

time (saline immediate spin technique, Sal-IS) during the $1 \mathrm{~W}$ period, centrifuged, resuspended and examined for agglutination. To each tube of saline method, 2 drops of 20\% polyethylene-glycol (PEG) (prepared in house)[6] were added as an enhancer and incubated at $37^{\circ} \mathrm{C}$ for 15 minutes, washed four times with saline, and 2 drops of anti-human IgG reagent (Ortho Anti-IgG (Rabbit), Ortho Clinical Diagnostics) were added. After incubation and centrifugation, the tubes were examined for agglutination. The in-house IgG-coated RBCs were added to each negative tube for confirmation of negative agglutination.

During the $2 \mathrm{~W}$ period, screening was performed by a combination of Sal-RT10', Bromelain one-step (Bro), and PEG-potentiated indirect antiglobulin test (PEG-IAT). In Bromelain one-step technique, 2 drops of patient's serum/plasma, 1 drop of Bromelain reagent (Wako Pure Chemical Industries, Osaka, Japan) were mixed and incubated for 15 minutes at $37^{\circ} \mathrm{C}$, and agglutination was determined after centrifugation. Screening for the $1 \mathrm{~W}$ cohort was done by Sal-IS and PEG-IAT methods.

Resolve Panels A and B (Ortho Clinical Diagnostics) and/or PANOCELL-16 (Immucor Gamma, GA, USA) were used for antibody identification.

We did not omit direct cross-matching, even when screening did not detect unexpected alloantibodies. Samples collected within 3 days of RBC transfusion for patients ever transfused or pregnant within 3 months were used for cross-matching by albumin-enhanced indirect antiglobulin testing (ALB-IAT) through June, 2004, and with low ionic strength solution-enhanced indirect antiglobulin testing (LISS-IAT) since July, 2004.

\section{Clinical diagnosis of DHTR}

DHTR was diagnosed per Serious Hazards of Transfusion (SHOT) criteria [8] and Ness et al. [9] when hemolytic findings included positive direct 


\section{EDORIUM Journals}

antiglobulin test (DAT), symptoms of jaundice, decreased hemoglobin, kidney dysfunction, plus at least one of the followings confirmed between 24 hours and 90 days after transfusion: (i) a new antibody or increase in the titers of pre-existing antibodies; (ii) antibody elution from transfused red blood cells.

\section{Statistical analysis}

Statistical analysis was performed using the Chisquared test (with or without Yates' correction), with $\mathrm{p}<0.05$ regarded as statistically significant. The $95 \%$ confidence interval (95\% CI) was used to compare antibody detection rates between the 2 groups and the frequency of DHTR. StatMate IV for Windows (ATMS, Tokyo) was used for analysis.

\section{RESULTS}

\section{Alloantibody detection rate and anti- body specificity}

Alloantibodies were detected in $1.22 \%$ of cases within $2 \mathrm{~W}$, and $1.33 \%$ of cases within $1 \mathrm{~W}$, with no statistically significant difference. As shown in Table 1, alloantibody specificity differed between the two groups, with an anti-Fy ${ }^{\mathrm{b}}$ detection frequency within $1 \mathrm{~W}$, significantly higher than that of within $2 \mathrm{~W}(\mathrm{p}<0.001)$. Anti-Di ${ }^{\mathrm{a}}$ also showed a similar trend, but without achieving statistical significance $(\mathrm{p}=0.065)$. Detection rates of anti-c, anti-P1, and anti-Le ${ }^{\mathrm{a}}$ were statistically lower within $1 \mathrm{~W}$ versus $2 \mathrm{~W}$.

Table 1: Antibody detection rate and specificity detected) by screening within 2 weeks (2W) and within 1 week (1W) of transfusion.

\begin{tabular}{|c|c|c|c|c|c|c|}
\hline \multirow{3}{*}{\multicolumn{2}{|c|}{$\begin{array}{l}\text { Number of patients } \\
\text { Number of cases in which antibody is } \\
\text { detected }\end{array}$}} & \multicolumn{2}{|c|}{ Within 2 weeks period } & \multicolumn{2}{|c|}{ Within 1 week period } & \multirow[t]{2}{*}{ P value } \\
\hline & & \multicolumn{2}{|c|}{32,601} & \multicolumn{2}{|c|}{44,896} & \\
\hline & & \multicolumn{2}{|c|}{398} & \multicolumn{2}{|c|}{595} & \\
\hline \multicolumn{2}{|l|}{$\%[95 \% \mathrm{CI}]$} & \multicolumn{2}{|c|}{$1.22[1.10-1.34]$} & \multicolumn{2}{|c|}{$1.33[1.22-1.43]$} & NS \\
\hline Antibody speci & group system & No. & $(/ 100,000)$ & No. & $(/ 100,000)$ & \\
\hline \multirow{6}{*}{ Rh system } & $\mathrm{D}$ & 14 & $(42.9)$ & 14 & $(31.2)$ & NS \\
\hline & $\mathrm{C}$ & 9 & $(27.6)$ & 20 & $(44.6)$ & NS \\
\hline & $\mathrm{c}$ & 33 & (101.2) & 23 & $(51.2)$ & $<0.05$ \\
\hline & $\mathrm{E}$ & 158 & $(484.7)$ & 235 & $(523.4)$ & NS \\
\hline & e & 7 & $(21.5)$ & 11 & $(24.5)$ & NS \\
\hline & others & 2 & $(6.1)$ & 1 & $(2.2)$ & NS \\
\hline \multirow{2}{*}{ Duffy system } & $\mathrm{Fy}^{\mathrm{a}}$ & o & $\mathrm{O}$ & o & o & - \\
\hline & $\mathrm{Fy}^{\mathrm{b}}$ & 13 & (39.9) & 52 & $(115.8)$ & $<0.001$ \\
\hline \multirow{2}{*}{ Kidd system } & $\mathrm{Jk}^{\mathrm{a}}$ & 20 & $(61.4)$ & 22 & $(49.0)$ & NS \\
\hline & $\mathrm{Jk}^{\mathrm{b}}$ & 4 & (12.3) & 4 & (8.9) & NS \\
\hline \multirow{2}{*}{ Kell system } & K & o & (o) & 3 & $(6.7)$ & NS \\
\hline & others & 2 & $(6.1)$ & 2 & $(4.5)$ & NS \\
\hline \multirow{2}{*}{ Diego system } & $\mathrm{Di}^{\mathrm{a}}$ & 20 & $(61.4)$ & 45 & $(100.2)$ & NS(0.065) \\
\hline & $\mathrm{Di}^{\mathrm{b}}$ & o & o & 1 & $(2.2)$ & NS \\
\hline P system & $\mathrm{P} 1$ & 24 & $(73.6)$ & 8 & $(17.8)$ & $<0.001$ \\
\hline \multirow{5}{*}{ MNS system } & M & 18 & $(55.2)$ & 30 & $(66.8)$ & NS \\
\hline & $\mathrm{N}$ & o & $\mathrm{o}$ & o & o & - \\
\hline & $\mathrm{S}$ & 8 & $(24.5)$ & 21 & $(46.8)$ & NS \\
\hline & $\mathrm{s}$ & o & o & o & $\mathrm{o}$ & - \\
\hline & others & O & o & O & o & - \\
\hline \multirow{2}{*}{ Lewis system } & $\mathrm{Le}^{\mathrm{a}}$ & 112 & $(343.6)$ & 111 & $(247.2)$ & $<0.05$ \\
\hline & $\mathrm{Le}^{\mathrm{b}}$ & 4 & $(12.3)$ & 10 & $(22.3)$ & NS \\
\hline Other system & & 12 & $(36.8)$ & 17 & (37.9) & NS \\
\hline Not identified & & 3 & $(9.2)$ & 1 & $(2.2)$ & NS \\
\hline Total & & 463 & & 631 & & \\
\hline
\end{tabular}

NS: Not significant 


\section{EDORIUM Journals}

Int J Blood Transfus Immunohematol 2018;8:100042Z02NT2018. www.ijbti.com

\section{Comparison of frequency of DHTR}

DHTRs occurred in 8 (0.13\%, or 1 in 779) of 6,234 recipients within $2 \mathrm{~W}$, but only 2 (0.02\%, or 1 in 4,031$)$ of 8,066 recipients within $1 \mathrm{~W}(\mathrm{p}<0.05)$, as shown in Table 2. One within $2 \mathrm{~W}$ developed an acute hemolytic reaction attributed to anti- $\mathrm{Bg}^{\mathrm{a}}+\mathrm{Bg}^{\mathrm{c}}$, as well as one DHTR [10]. Thus, the overall incidence of hemolytic transfusion reactions was $0.14 \%(9 / 6,234)$ among within $2 \mathrm{~W}$ and $0.02 \%(2 / 8,066)$ among within $1 \mathrm{~W}(\mathrm{p}=0.02)$.

Table 3 shows the antibody specificity and the days from transfusion to hemolytic transfusion reaction for each case. The causative antibodies for hemolytic transfusion reactions were $\mathrm{Rh}$ in 5 , Kidd in $3, \mathrm{Rh}+$
Kidd in 2, and Bg in 1 cases. All of these patients had a history of transfusion or pregnancy. The time between RBC transfusion and DHTR (excluding acute hemolysis) ranged from 7 to 39 days.

\section{DISCUSSION}

There are insufficient data about the timing of alloantibodies formation and detection following red cell transfusion. Rarely, new antibodies are detected within the first 3 days, e.g. $0.4 \%$ (11 of 2,932 cases) or $2.3 \%$ of all immunized patients including secondary immune response [11]. Moreover, the majority (83\%) of new

Table 2: Frequency of delayed hemolytic transfusion reactions among cohorts screened within 2 weeks (2W) and within 1 week (1W) of transfusion.

\begin{tabular}{|c|c|c|c|}
\hline & $\mathbf{2 W}$ & $1 \mathrm{~W}$ & p value \\
\hline Number of red cell transfused patients & 6,234 & 8,066 & \\
\hline $\begin{array}{l}\text { Number of patients with delayed hemolytic } \\
\text { transfusion reaction }\end{array}$ & 8 & 2 & \\
\hline$\%[95 \% \mathrm{CI}]$ & $\begin{array}{c}0.13[0.04-0.22] \\
(1 \text { in } 779)\end{array}$ & $\begin{array}{l}0.02[0.00-0.06] \\
\quad(1 \text { in } 4,031)\end{array}$ & 0.045 \\
\hline Number of hemolytic transfusion reactions & $9^{*}$ & 2 & \\
\hline$\%[95 \% \mathrm{CI}]$ & $0.14[0.05-0.24]$ & $0.02[0.00-0.06]$ & 0.024 \\
\hline
\end{tabular}

*Including one acute hemolytic transfusion reaction by anti-Bga $+\mathrm{Bg}^{\mathrm{c}}$ (reference 10)

Table 3: Antibody specificity and post-transfusion period until hemolytic transfusion reaction

\begin{tabular}{|c|c|c|c|c|c|c|c|c|}
\hline $\begin{array}{c}\text { Case } \\
\text { no. }\end{array}$ & $\operatorname{Sex}$ & $\begin{array}{c}\text { Age } \\
\text { (years) }\end{array}$ & $\begin{array}{c}2 W / 1 W \\
\text { Screening } \\
\text { interval }\end{array}$ & Antibody found & $\begin{array}{c}\text { Prior } \\
\text { transfusion } \\
\text { history }\end{array}$ & $\begin{array}{c}\text { Prior } \\
\text { pregnancy } \\
\text { history }\end{array}$ & $\begin{array}{l}\text { Post- } \\
\text { transfusion } \\
\text { days until } \\
\text { hemolysis }\end{array}$ & $\begin{array}{c}\text { Cross } \\
\text { match } \\
\text { test }\end{array}$ \\
\hline $1^{*}$ & $\mathrm{~F}$ & 43 & $2 \mathrm{~W}$ & anti-E+ autoantibody & yes & yes & 8 & ALB-IAT \\
\hline 2 & $\mathrm{~F}$ & 77 & $2 \mathrm{~W}$ & anti-E+ autoantibody & no & yes & 39 & LISS-IAT \\
\hline 3 & $\mathrm{~F}$ & 75 & $2 \mathrm{~W}$ & anti-E & yes & yes & 27 & ALB-IAT \\
\hline 4 & M & 22 & $2 \mathrm{~W}$ & anti-E+c & yes & no & 16 & ALB-IAT \\
\hline 5 & $\mathrm{~F}$ & 36 & $2 \mathrm{~W}$ & anti-E & yes & yes & 8 & ALB-IAT \\
\hline $6-1$ & & & $2 \mathrm{~W}$ & anti-E+c+Jk ${ }^{\mathrm{a}}$ & yes & yes & 14 & ALB-IAT \\
\hline $6-2^{* *}$ & $\mathrm{~F}$ & 77 & $2 \mathrm{~W}$ & anti-Bga $+B g^{c}$ & & & 0.3 & ALB-IAT \\
\hline 7 & M & 16 & $2 \mathrm{~W}$ & anti-Jk ${ }^{\mathrm{a}}$ & yes & no & 10 & ALB-IAT \\
\hline 8 & $\mathrm{~F}$ & 71 & $2 \mathrm{~W}$ & anti-Jk ${ }^{\mathrm{a}}$ & yes & yes & 17 & LISS-IAT \\
\hline $9^{*}$ & $\mathrm{~F}$ & 55 & $1 \mathrm{~W}$ & $\begin{array}{l}\text { Anti-Jk }{ }^{\mathrm{a}}+\mathrm{E}+\mathrm{c} \\
+\mathrm{S}+\mathrm{Bg}^{\mathrm{a}}+ \\
\text { autoantibody }\end{array}$ & yes & yes & 11 & LISS-IAT \\
\hline 10 & M & 60 & $1 \mathrm{~W}$ & anti-Jk ${ }^{\mathrm{a}}$ & yes & no & 7 & LISS-IAT \\
\hline
\end{tabular}

ALB-IAT: Albumin-indirect antiglobulin test

LISS-IAT: Low ionic strength solution-indirect antiglobulin test

* Delayed hemolytic transfusion reaction plus hyperhemolysis

** Acute hemolytic transfusion reaction by anti-Bga+Bgc (reference 10) 


\section{EDORIUM Journals}

antibodies are detected after more than 14 days, whereas only $17 \%$ are detected within 14 days after transfusion [11]. It is better established that most new antibodies manifest within the next 30 days, and very few antibodies develop after three months [3].

Japan's National Health Insurance system has, since April 2006, agreed to cover alloantibody screening up to four times per month, versus twice per month prior to transfusion for patients who may have possibly transfusion. In order to investigate whether there are any beneficial effects of more frequent screening, with respect to alloantibody detection and frequency of DHTR, this retrospective, single-center, observational study was performed. In 2016, the Japan Society of Blood Transfusion and Cell Therapy advised that patients previously transfused or pregnant within the previous 3 months, should be cross-matched using samples no more than 3 days old at the time of transfusion [12].

Among clinically significant antibodies, we observed that the detection rate of anti-Fyb increased clearly and anti-Dia tended to increase within $1 \mathrm{~W}$ versus $2 \mathrm{~W}$, probably because screening was done closer to the time of transfusion. It should be noted that PEG-IAT, reported to be more sensitive than other techniques, especially in detecting Kidd, Duffy, and Rh system antibodies [2], has been used as a standard screening test throughout both periods [6].

On the other hand, the detection frequency of anti-c decreased significantly from 101.2 within $2 \mathrm{~W}$ to 51.2 per 100,000 within $1 \mathrm{~W}$ ( $\mathrm{p}<0.05$ ). Anti-c is usually codetected with anti-E, i.e. anti-E + c among Japanese, rather than emerging as a single antibody. This decreased anti-c frequency may be due to earlier detection of anti-E by a shortened sampling period and earlier selection of E-compatible RBCs for transfusion. Early anti-E detection within three days after transfusion has been observed in a previous study [11]. Among Japanese, the phenotype $\mathrm{Rh}$ Dee has a prevalence of 50\%, consisting of DCCee at $43 \%$ and DCcee at $7 \%$ [13]. Therefore, when one bag of E-compatible RBCs is selected, $86 \%$ of the time it will lack c antigen; this serves as an incidental prophylaxis against the development of anti-c.

Of note, the detection rates of cold antibodies, anti$\mathrm{Le}^{\mathrm{a}}$ and anti-P1, significantly decreased ( $\mathrm{p}<0.01, \mathrm{p}$ $<$ o.001). Because we changed screening from Sal-RT10' plus Bro and PEG-IAT within 2W to Sal-IS and PEG-IAT within $1 \mathrm{~W}$, these cold-reactive antibodies were less often detected. When anti-Le ${ }^{\mathrm{a}}$ and anti-P1 results are negative with a potentiator-free indirect anti-globulin test, it has been suggested that these antigens can be ignored when selecting “compatible” RBCs [3, 4, 14]

DHTRs observed in our study decreased significantly within $1 \mathrm{~W}$ compared with $2 \mathrm{~W}(\mathrm{p}=0.045)$. In the past, DHTRs due to a primary immune response were often reported $[15,16]$, but since then DHTRs have shifted predominantly to secondary immune responses $[17$, 18]. This shift might be generally attributed to technical changes, including the allowed sample collection period and test sensitivity. Also in this study, all patients who developed DHTRs had past transfusion and/or pregnancy history, which is compatible with a concept that many DHTRs can arise from a secondary immune response. Even if an antibody was previously detected after a past transfusion or pregnancy but falls below detection sensitivity in a current screening test, incompatible RBCs could evoke a DHTR. In our experience, the time until DHTR ranged from 7 to 39 days; therefore, with a 2 week screening interval, alloantibodies can be missed and incompatible RBCs might be administered. Nevertheless, while DHTRs are not completely preventable, by screening with the most sensitive methods such as PEG-IAT [2, 6] using samples drawn within one week of transfusion and cross-matching with samples drawn within three days of transfusion, DHTRs are decreasing.

In the UK, where the vast majority of laboratories report through SHOT, a total of 18 cases of DHTR, including hyperhaemolysis, were documented in 2016, a number substantially lower than reported in previous years: 28 in 2015 and 2014, 32 in 2013, and 33 in 2012 [8]. Although approximately $80 \%$ are transfused within 72 hours of a new sample for screening and crossmatching being taken [19], BCSH speculates that this does not increase the number of additional DHTR cases being reported [3].

\section{CONCLUSION}

In conclusion with the decrease in the time allowed to collect alloantibody screening samples from within two weeks to within one week of transfusion, clinically significant antibody detection selectively increased while the frequency of DHTR decreased.

\section{REFERENCES}

1. Stack G, Tormey CA. Detection rate of blood group alloimmunization based on real-world testing practices and kinetics of antibody induction and evanescence. Transfusion 2016 Nov;56(11):2662-7.

2. Pineda AA, Vamvakas EC, Gorden LD, Winters JL, Moore SB. Trends in the incidence of delayed hemolytic and delayed serologic transfusion reactions. Transfusion 1999 Oct;39(10):1097-103.

3. Milkins C, Berryman J, Cantwell C, et al. Guidelines for pre-transfusion compatibility procedures in blood transfusion laboratories. British Committee for Standards in Haematology. Transfus Med 2013 Feb;23(1):3-35.

4. Downes KA, Shulman IA. Pretransfusion Testing. In: Roback JD, Grossman BJ, Harris T, Hiller CD, editors. Technical Manual. 17ed. Bethesda: AABB; 2011. p. 437-62.

5. de Vries R, Haas F; working group for revision of the Dutch blood transfusion guideline 2011. English 


\section{EDORIUM Journals}

translation of the Dutch blood transfusion guideline 2011. Vox Sang 2012 Nov;103(4):363.

6. Okutsu M, Ohto H, Yasuda H, et al. Increased detection of clinically significant antibodies and decreased incidence of delayed haemolytic transfusion reaction with the indirect antiglobulin test potentiated by polyethylene glycol compared to albumin: A Japanese study. Blood Transfus $2011 \mathrm{Jul} ; 9(3): 311-9$.

7. Ono T, Ohto H, Yasuda H, et al. Comparative study of two automated pre-transfusion testing systems (microplate and gel column methods) with standard tube technique. Int $\mathrm{J}$ Blood Transfus Immunohematol 2017;7:15-25.

8. SHOT annual report and summaries. 2016, 2015, 2014, 2013, and 2012. [Available at: https://www. shotuk.org/shot-reports/]

9. Ness PM, Shirey RS, Thoman SK, Buck SA. The differentiation of delayed serologic and delayed hemolytic transfusion reactions: Incidence, longterm serologic findings, and clinical significance. Transfusion 1990 Oct;30(8):688-93.

10. Takeuchi C, Ohto H, Miura S, Yasuda H, Ono S, Ogata T. Delayed and acute hemolytic transfusion reactions resulting from red cell antibodies and red cell-reactive HLA antibodies. Transfusion 2005 Dec;45(12):1925-9.

11. Schonewille $\mathrm{H}$, van de Watering LM, Loomans DS, Brand A. Red blood cell alloantibodies after transfusion: factors influencing incidence and specificity. Transfusion 2006 Feb;46(2):250-6.

12. Okuda M, Ishimaru K, Uchikawa M, et al. Guidelines for pre-transfusion compatibility procedures in blood transfusion laboratories in Japan. (2nd edition). [Artical in Japanese]. Japanese Journal of Transfusion and Cell Therapy 2016:62(6):651-63.

13. Uchikawa M. Rh blood group system. In: Toyama $H$, Shibata Y, Maeda H, Ohto H, editors. Transfusion Medicine. 3rd ed. Tokyo: Chugai Igakusha; 2004. p. 211-48.

14. Judd WJ. How I manage cold agglutinins. Transfusion 2006 Mar;46(3):324-6.

15. Ishimaru K, Tenman C, Fujiwara Y, Ohe M, Kota T, Ikeda H. A case of delayed hemolytic transfusion reaction probably caused by a primary immune response. [Artical in Japanese]. Japanese Journal of Transfusion Medicine 2004;50(6):768-73.

16. Kitazawa J, Inomata M, Kamata C, et al. A case of delayed hemolytic transfusion reaction due to anti$\mathrm{c}+\mathrm{e}$ antibody caused by primary immune response. [Artical in Japanese]. Japanese Journal of Transfusion Medicine 2005;51(6):594-600.

17. Kay B, Poisson JL, Tuma CW, Shulman IA. Anti$\mathrm{Jk}^{\mathrm{a}}$ that are detected by solid-phase red blood cell adherence but missed by gel testing can cause hemolytic transfusion reactions. Transfusion 2016 Dec;56(12):2973-9.

18. Sakuragi M, Kiyokawa T, Hosokawa M, et al. Clinical course of alloimmunized patients after red blood cell transfusion. [Artical in Japanese]. Japanese Journal of Transfusion and Cell Therapy 2013;59(4):579-85.

19. Milkins C, Berryman J, Cantwell C, et al. Timing of sample collection in relation to previous transfusions: A proposal for changing the recommendations. Transfus Med 2010;20:39.

\section{Author Contributions}

Nozomi Takano - Acquisition of data, Analysis and interpretation of data, Drafting the article, Revising it critically for important intellectual content, Final approval of the version to be published

Hiroyasu Yasuda - Acquisition of data, Analysis and interpretation of data, Drafting the article, Revising it critically for important intellectual content, Final approval of the version to be published

Masami Kikuchi - Acquisition of data, Drafting the article, Final approval of the version to be published

Kinuyo Kawabata - Acquisition of data, Analysis and interpretation of data, Drafting the article, Final approval of the version to be published

Chikako Takeuchi-Baba - Acquisition of data, Drafting the article, Final approval of the version to be published Satoshi Ono - Acquisition of data, Drafting the article, Final approval of the version to be published

Takako Ono - Acquisition of data, Drafting the article, Final approval of the version to be published

Keiji Minakawa - Acquisition of data, Analysis and interpretation of data, Drafting the article, Revising it critically for important intellectual content, Final approval of the version to be published

Akiko Sugawara - Acquisition of data, Drafting the article, Final approval of the version to be published

Saori Miura - Acquisition of data, Drafting the article, Final approval of the version to be published

Kazuya Watanabe - Acquisition of data, Drafting the article, Final approval of the version to be published

Maiko Abe - Acquisition of data, Drafting the article, Final approval of the version to be published

Hiroe Suzuki - Acquisition of data, Drafting the article, Final approval of the version to be published

Mao Watanabe - Acquisition of data, Drafting the article, Final approval of the version to be published

Mutsumi Sasaki - Acquisition of data, Drafting the article, Final approval of the version to be published Kazuhiko Ikeda - Acquisition of data, Analysis and interpretation of data, Drafting the article, Revising it critically for important intellectual content, Final approval of the version to be published

Kenneth E. Nollet - Acquisition of data, Drafting the article, Revising it critically for important intellectual content, Final approval of the version to be published Hitoshi Ohto - Substantial contributions to conception and design, Analysis and interpretation of data, Drafting the article, Revising it critically for important intellectual content, Final approval of the version to be published

\section{Guarantor of Submission}

The corresponding author is the guarantor of submission.

\section{Source of Support}

None 


\section{Consent Statement}

Written informed consent was obtained from the patient for publication of this original article.

\section{Conflict of Interest}

Authors declare no conflict of interest.

\section{Copyright}

(C) 2018 Nozomi Takano et al. This article is distributed under the terms of Creative Commons Attribution License which permits unrestricted use, distribution and reproduction in any medium provided the original author(s) and original publisher are properly credited. Please see the copyright policy on the journal website for more information.
Access full text article on other devices

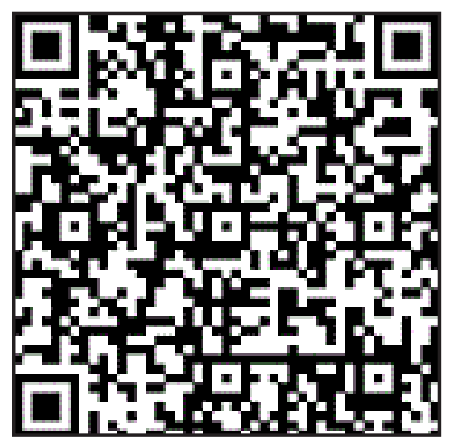

Access PDF of article on other devices

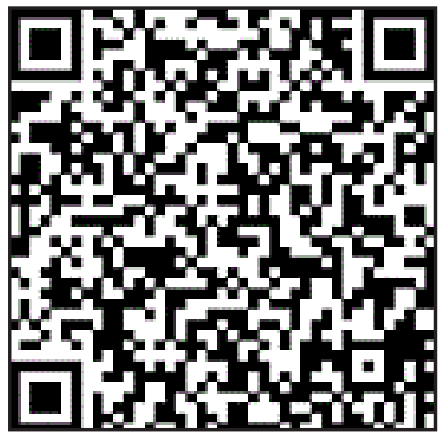

